\title{
La noción de constitución en el análisis de la experiencia perceptiva*
}

\author{
Olga FERnÁndEz PRAT \\ Departamento de Filosofía \\ Facultad de Filosofía y Letras \\ Universitat Autònoma de Barcelona \\ olga.fernandez@uab.es
}

\begin{abstract}
Resumen: En este artículo se pretende explicar en qué consiste el problema de la constitución en el marco de la experiencia perceptiva y defender su vigencia. Se muestra cómo el problema, de raíces kantianas, ha sido abordado en las principales tradiciones filosóficas del siglo Xx ejemplificándolo en tres figuras centrales: Husserl, Cassirer y Carnap, y que el tratamiento husserliano configura un nuevo escenario. Aunque en la actualidad el término 'constitución' prácticamente haya desaparecido de la filosofía contemporánea, apoyándome en las claves interpretativas que ofrezco pretendo mostrar que este hecho no significa que el mencionado problema no siga siendo un importante objeto de estudio en la filosofía de la percepción y, en general, de la mente.
\end{abstract}

Palabras clave: fenomenología, noema, filosofía analítica, modo de presentación

\begin{abstract}
In this paper I try to explain what the problem of constitution in the framework of perceptual experience consists in, and also to argue that this problem continues to be in force. Through three key philosophers - Husserl, Cassirer, and Carnap - it is shown how the problem, which has Kantian roots, has been treated in the main philosophical traditions of the twentieth century, and furthermore, how the Husserlian approach sets up a new scenario. Although at the present time the term 'constitution' has practically disappeared from the philosophical scene, on the basis of the interpretative clues here offered I try to show that this fact does not mean that the alluded problem does not continue to be a significant subject in the study of perception and the mind.
\end{abstract}

Key words: phenomenology, noema, analytic philosophy, mode of presentation

El problema de la constitución se presenta con la eclosión del representacionalismo en la filosofía del siglo XVII, aunque, propiamente, su articulación se encuentra por primera vez en la filosofía de Kant. Ya en el siglo XX este problema se plantea en las tres grandes tradiciones

*Este artículo ha sido financiado por el proyecto de investigación HUM200507539-C02-02. El haber podido rastrear en Carnap y Husserl la idea de constitución se lo debo a Daniel Quesada.

Diánoia, volumen LIII, número 60 (mayo 2008): pp. 141-165. 
filosóficas de su primer tercio: en la fenomenología, especialmente en Husserl; en el neokantismo, especialmente en Cassirer, y en lo que finalmente vendría a ser la tradición analítica, especialmente en Carnap. El problema, que afecta a tres áreas de la filosofía - la ontología, la epistemología y la filosofía de la mente-, desaparece posteriormente de la agenda filosófica por razones diversas, de las que nos ocuparemos sintéticamente. Sin embargo, como se argumentará, en realidad tiene plena vigencia, y el hecho de que no sea "visible" en el panorama filosófico actual se debe a que, por así decir, se presenta "camuflado". Previamente se defenderá que, en el tratamiento del problema de la constitución en el ámbito de la percepción, el punto de inflexión principal se encuentra en la fenomenología husserliana, pues, como se verá, es finalmente ésta la perspectiva bajo la cual puede mostrarse la vigencia del problema.

En la primera sección se explicará de un modo preliminar cuál es el problema de la constitución, y en la última se mostrará por qué ha de considerarse vigente el problema. En las tres secciones intermedias, el problema de la constitución se presenta, respectivamente, en el contexto de las tradiciones kantiana y neokantiana, analítica (Carnap) y fenomenológica (Husserl). Es necesario realizar con cierto detenimiento este recorrido histórico por varias razones. Ante todo, hay que tener en cuenta que la noción relevante de constitución no se presta bien a una caracterización general o abstracta, y se precisa examinar los diversos contextos filosóficos en los que aparece para asegurarse de que no son meras convenciones terminológicas las responsables de que agrupemos ciertas cuestiones filosóficas bajo un rótulo común, sino que está justificado ver que en esos contextos existe una problemática común bajo diversas formas. Pero, además, el intento de quitar la pátina que con el tiempo se ha acumulado sobre esa problemática tiene un interés propiamente filosófico si, como aquí se defenderá, es cierto que el problema de la constitución sigue vigente, pues esa historia pone entonces de manifiesto cuánto hay de superficial o artificioso en la separación de corrientes filosóficas que en la actualidad parecen habitar mundos distintos.

\section{1. ¿Cuál es el problema de la constitución?}

El problema de la constitución se plantea cuando se sostiene que aquello de lo que se es inmediatamente consciente - por ejemplo, y especialmente, en la percepción- no son objetos del dominio público, como puedan serlo los objetos materiales, sino entidades subjetivas. Bajo el supuesto de que, finalmente, la percepción nos pone de algún modo en 
contacto con los mismos objetos (los mismos en un sentido intersubjetivo), y dado que, según es ampliamente reconocido, el conocimiento, especialmente el conocimiento científico, es intersubjetivamente compartido - acerca de algo compartido-, se trata de explicar cómo es esto posible. Una posibilidad, en principio, es considerar que los objetos del dominio público están "constituidos" de alguna manera por o a partir de las entidades subjetivas inmediatamente accesibles a la mente. El problema es, entonces, explicar en qué consiste esa constitución.

Siendo el problema de la constitución el que es, se presenta históricamente, al menos en forma latente, cuando aparecen las teorías representacionalistas de la percepción; sin embargo, se muestra con particular fuerza en Kant y en buena parte de la filosofía que se desarrolla bajo su influencia. Como es sabido, para Kant, debemos rechazar que los objetos de la percepción y el conocimiento - aquellos sobre los que versan nuestros juicios verdaderos- sean objetos que transcienden nuestra experiencia sensorial —unos objetos que están "tras" ella—, ni pueden, desde luego, ser esos objetos las entidades no conceptualizadas que según los empiristas constituían lo dado a la conciencia. En el primer caso, puesto que —según Kant- no tenemos acceso a tales entidades, serían imposibles los juicios verdaderos y el conocimiento. En el segundo lo serían igualmente, aunque por una razón distinta: la corriente de la experiencia sensorial "desnuda" es completamente caótica e indiferenciada. Así, los objetos de nuestros juicios (incluidos los juicios de percepción) y del conocimiento no existen con independencia de nuestra capacidad de formular tales juicios, sino que son "constituidos" cuando los datos sensoriales no conceptualizados se enmarcan $\mathrm{u}$ organizan dentro de las estructuras a priori que hacen posible el juicio.

Como puede verse, en el marco kantiano la constitución afecta directamente, por así decir, a los objetos de los juicios y el conocimiento. Sin embargo, el problema experimenta un giro crucial cuando se articulan explícitamente teorías de la intencionalidad y se defiende además -como ocurre con Husserl- que la intencionalidad no es, por así decir, cosa de dos (sujeto y objeto), sino que involucra un tercer elemento. Al menos esto es lo que se defiende en el presente trabajo.

2. Constitución del objeto de la percepción y constitución del objeto del conocimiento empírico: Kant y el neokantismo

En su revelador libro A Parting of the Ways (2000), Michael Friedman rastrea las raíces kantianas de la filosofía contemporánea centrándose especialmente en tres grandes figuras de la filosofía del siglo Xx: Cassi- 
rer, Heidegger y Carnap. La presente sección y las dos siguientes comparten el espíritu de esa obra de Friedman con la importante diferencia de que, en lugar de centrarse - como en ella ocurre- en la cuestión del lugar de la lógica en la filosofía, se ocupa de la cuestión de la constitución — sólo de paso mencionada en aquella obra-, circunscrita sobre todo al ámbito de la percepción. El efecto más inmediato es que es imprescindible tratar la contribución de Husserl —que ocupa un lugar relativamente menor en el libro de Friedman-, lo que comporta tener que enfocar con nitidez una tradición filosófica distinta de la kantiana. En contraposición, se puede dejar de lado la figura de Heidegger, en cuya filosofía la percepción ocupa - por razones teóricas que no vienen al caso- un lugar menor.

Consideremos, para empezar, un ejemplo sencillo con el fin de plantear un aspecto central del problema que nos ocupará. Supongamos que dirigimos nuestra mirada intencionadamente a la puerta de una casa y que vemos allí sentado a un perro. Lo que hemos percibido, podríamos decir, es un animal, más precisamente un perro. Esta descripción del estado de percepción es, obviamente, una descripción según la cual el objeto percibido se describe utilizando un concepto (perro). ¿Es adecuado describir así nuestra percepción visual? Si en algún sentido lo es, ¿en qué sentido exactamente?

Nótese que no planteamos el problema de si es adecuado describir aproximadamente del modo indicado el juicio que sobre la base de nuestra mirada podríamos haber hecho sobre lo que está ahí sentado en la puerta de esa casa. Alguien que posea el concepto de perro y en quien se cumplan las condiciones adecuadas - ambientales (como luz suficiente) o personales (como suficientemente buena vista) - puede sin duda hacer ese juicio y aceptarlo, formando la creencia perceptiva correspondiente. Pero nosotros indagamos por la experiencia perceptiva misma y quisiéramos, si es posible, permanecer neutrales acerca de la cuestión de si, de algún modo, el juicio forma parte de la experiencia perceptiva.

Al tratar de sintetizar lo que sobre el problema puede decirse desde la filosofía kantiana, es preciso advertir enseguida que no se encuentra en Kant una doctrina de la percepción como una unidad temática, sino que es preciso buscarla realizando un ejercicio de reconstrucción que requiere entrar en delicadas cuestiones de interpretación sobre multitud de temas y tesis kantianas (como la sensibilidad y sus formas a priori, el papel de la imaginación, el contraste entre formas de la intuición e intuiciones formales, la doctrina de las categorías, la síntesis de la "apercepción" y la relación de la imaginación con las ca- 
tegorías) que configuran lo que en su obra son propiamente unidades temáticas (como la Deducción Trascendental de las categorías), las cuales, a su vez, ya están sujetas a muy delicados problemas de interpretación.

Puesto que Kant separa tajantemente las facultades de la sensibilidad y el entendimiento, se podría ingenuamente pensar en una división que reflejara directamente esa distinción de facultades: el objeto de la percepción sería el objeto no conceptualizado configurado en la primera facultad (el contenido referencial de una intuición kantiana constituida sólo por el espacio y el tiempo en lo que respecta a los elementos que el sujeto aporta) y el objeto o contenido conceptual del juicio y el conocimiento sería algo configurado ulteriormente por la segunda. Sin embargo, la doctrina kantiana está en realidad muy alejada de esta simple división y se caracteriza de un modo general por la polaridad subjetivo-objetivo: la subjetividad de la percepción frente a la objetividad del juicio verdadero o el conocimiento.

En un conocido ensayo, Strawson, tratando de aportar alguna luz - desde una perspectiva hasta cierto punto wittgensteiniana- sobre el papel que, según su interpretación de Kant, los conceptos desempeñan en la percepción, recurre a una serie de metáforas: "la experiencia visual está irradiada por, infusa de (infused with), el concepto; o se empapa del concepto" (Strawson 1970, p. 57), que sustituirían —de un modo más preciso, cabe decir- a la del propio Wittgenstein (el ver como "eco" del pensamiento, Philosophical Investigations, p. 212; p. 485 de la versión al español). Estas metáforas sugieren, desde luego, la concepción según la cual el contenido de la experiencia visual misma es un contenido conceptual. Strawson mismo es un defensor de ese punto de vista, desarrollado posteriormente en direcciones innovadoras por McDowell; pero la cuestión de si hay que atribuírselo al propio Kant (y, en verdad, la de si necesariamente la descripción metafórica lo implica lógicamente) es, cuando menos, controvertida.

El problema es el de si, para Kant, el contenido de la experiencia visual (en el ejemplo, por lo que respecta a la parte, podríamos decir, "perruna" de ella) es el mismo que el del juicio correspondiente. Sellars sostuvo con fuerza la necesidad de separar ambos. En Ciencia y metafísica formuló esta idea atribuyendo a Kant la posición según la cual el contenido de la experiencia es sólo "mínimamente conceptual", frente al carácter plenamente conceptual del contenido del juicio (cfr. Sellars 1968, pp. 1-30). Sin embargo, en "The Role of Imagination in Kant's Theory of Experience" pone el énfasis en el carácter perspectivista (Sellars 1978, pp, 237-240; Sellars atribuye a Kant el reconocimiento de 
ese carácter, al mismo tiempo que lo hace propio). Lo que esto último quiere decir es que en lo percibido contamos necesariamente con aspectos que no se nos presentan o que no nos son "dados". Young (1988) expresa esta idea paradójicamente cuando sostiene que en un estado de percepción se toma algo como "distinto o más de lo que se percibe que es".

En todo caso, este ir más alla de lo estrictamente presentado refleja la función esencial que Kant atribuye a la imaginación en la percepción, un papel funcional que, para muchos estudiosos de Kant -entre ellos, todos los mencionados-, se ha de concebir como interpretativa: la imaginación realiza una interpretación de la multiplicidad de la sensación. Esta capacidad, que al parecer incluye la capacidad de formar imágenes, pero no se limita a ella, es una capacidad de "síntesis imaginativa" gobernada por reglas. ${ }^{1}$ La cuestión de si Kant concibe el contenido de un estado de percepción como conceptual pasa necesariamente por tratar el papel de la imaginación en relación con el entendimiento y las categorías, algo que podemos hacer aquí sólo muy sucintamente. ${ }^{2}$

Así pues, de acuerdo con Kant, la percepción de, por ejemplo, un perro - que ha de distinguirse del juicio de que lo percibido es un perro o

${ }^{1}$ Young (1988) introduce la distinción crucial entre proceder de acuerdo con una regla o ley y proceder de acuerdo con una concepción de una regla o ley. Según él, para Kant la imaginación procedería del primer modo y el entendimiento del segundo. Véase también Allison 2004, pp. 186-189, en relación con estos temas. Allison critica aquí a Strawson por asociar el papel de la imaginación con la capacidad de formar imágenes, y elogia a Sellars por distinguir entre "imaging" (el formar imágenes) e "imagining". Pero, como el propio Allison menciona, hay evidencia textual directa en Kant de que éste asociaba el papel de la imaginación con el de formar imágenes (cfr. Crítica de la razón pura, A120-121). Quizá se podría aducir también el pasaje en que Kant da el ejemplo del esquema correspondiente al concepto de perro (aunque, en lo que es probablemente un lapsus terminológico — cfr. Allison, 2004, p. 208 — se dice que es el concepto): "una regla de acuerdo con la cual mi imaginación puede especificar la forma de un animal de cuatro patas en general, sin verse restringida a ninguna forma particular que me ofrezca la experiencia o a ninguna posible imagen que pueda exhibir en concreto". Aquí no parece que se pretenda excluir que el papel de la imaginación incluya el de generar imágenes, sino que se enfatiza que, en todo caso, no se limita a un grupo determinado de ellas. Sería, quizá, más prudente sostener que, para Kant, la imaginación no es una capacidad limitada a la formación de imágenes, y quizá ni siquiera principalmente una capacidad de producirlas (lo que resulta coincidir con una de las formulaciones del propio Allison).

${ }^{2}$ Sigo a Allison (2004) en estas líneas; cfr. capítulo 7, III C, y, muy en particular las pp. 196-197.

Diánoia, vol. LIII, no. 60 (mayo 2008). 
de cualquier juicio sobre el mismo- involucra a la imaginación, la cual realiza una "síntesis figurativa". En esta síntesis intervienen, además de las cualidades sensibles del perro captadas en la sensación (principalmente el color), propiedades espaciales como una cierta forma y tamaño (también hay necesariamente una dimensión temporal, que dejamos de lado para simplificar). Estas determinaciones espaciales presuponen una concepción del espacio como unitario y homogéneo, lo que requiere las categorías (en la Crítica de la razón pura, B160-161, Kant argumenta sucintamente que debido, por así decir, a la intervención del espacio y el tiempo, toda percepción está sujeta a las categorías). Pero, al tratarse de una percepción y no de un juicio, la forma en que invervienen las categorías no consiste en que el objeto quede subsumido bajo una categoría (el perro bajo la categoría de sustancia), sino que al menos una de las categorías interviene en la regla misma a la que la síntesis está sujeta. La síntesis en que consiste la percepción del objeto (el perro), tratado tridimensionalmente por la imaginación, tiene un aspecto formal: está condicionada por la determinación del espacio que se percibe que éste ocupa; es decir, se ve sujeta a las condiciones de determinación de ese espacio. Así, la regla que concreta este condicionamiento debe "estar de acuerdo" con la categoría de cantidad (B162). ${ }^{3}$

De todo este intrincado asunto, que forma parte del núcleo mismo de la Deducción Trascendental en la Crítica, en lo que es importante insistir para nuestros propósitos aquí es que, como hemos visto, para Kant las categorías (o al menos alguna de ellas) están involucradas no solamente en la formación de juicios, sino en el proceso de percepción en cuanto "apercepción empírica"; es decir, en cuanto apercibimiento inmediato de los contenidos de la conciencia resultado de un episodio de percepción (ésta sería la tesis central de $\S 26$ en la Deducción Trascendental). Así, el objeto o contenido de la percepción está constituido por elementos pertenecientes no sólo a la facultad de la sensibilidad, sino también a la del entendimiento, y sin embargo, por otra parte, hay que distinguirlo de los contenidos - contenidos de conceptos, esquemas y juicios- que propiamente configuran esta última.

De este modo, si bien, como se ha advertido anteriormente, no hay en Kant una doctrina de la percepción como unidad temática, sí que

${ }^{3}$ Que el aspecto del espacio que aquí interviene — se trata de un espacio determinado y, por lo tanto, de una intuición formal, no meramente una forma de la intuición - requiere una unidad sintética se argumenta en una nota a B160-161 y que todo lo que está sujeto a esta unidad requiere las categorías se argumenta previamente en B143; cfr. Allison 2004, pp. 191-192 y 177, respectivamente. 
existe una base sólida para distinguir entre el objeto de la percepción y el objeto del conocimiento empírico. Sin duda, en claro contraste con el tratamiento del primero, su análisis del segundo es un tema específico y centralmente kantiano, algo que concierne a la experiencia objetiva o experiencia con validez objetiva (Erfahrung, en la terminología de Kant). Con todo, la primera temática no queda completamente absorbida por la segunda, por decirlo así, dada la separación entre las facultades de la sensibilidad y el entendimiento.

Es justo este obstáculo a la completa "fagocitación" de la primera temática por la segunda el que desaparece en las escuelas neokantianas de finales del siglo XIX y principios del siglo XX -notablemente las llamadas Escuela de Marburgo y la Escuela del Sudoeste (de Alemania)—, ya que es común a ellas el rechazo de la dualidad kantiana de facultades. Con ello, los elementos subjetivos (en el sentido de "dependientes del sujeto humano") en la constitución de la experiencia se recogen en la "capacidad generadora del pensamiento", en la que quedan integrados los aspectos espacio-temporales que constituían en Kant el dominio de una facultad separada de la sensibilidad. ${ }^{4}$ Como consecuencia, la concepción kantiana sobre los objetos de percepción y conocimiento sufre una profunda transformación, que pasamos a examinar brevemente a continuación, centrándonos, entre las diversas versiones, correspondientes a las variantes del neokantismo, en la importante línea NatorpCassirer, especialmente tal como la desarrolló este último en Sustancia y función (Cassirer 1910).

La filosofía de Cassirer se caracteriza por su oposición radical a los dualismos epistemológicos y metafísicos. En particular, se opone a la dualidad subjetivo-objetivo también en el dominio de la percepción. Lo subjetivo y lo objetivo en la percepción son -como en cualquiera otra de las dualidades- "términos correlativos"; es decir, términos únicamente caracterizables en su relación mutua, y por ello aplicables de modo cambiante a aquello a lo que se aplican. Así, si atendemos a aquello de lo que somos conscientes en nuestra experiencia perceptual ordinaria - un color, una forma espacial — podríamos inclinarnos a caracterizar de objetivo el contenido de esa experiencia en virtud de su carácter de "inmediatamente dado" ${ }^{5} \mathrm{y}$, sin embargo, el criterio de permanencia, repetibilidad y acuerdo intersubjetivo pronto nos hace inclinarnos por diferenciar la impresión subjetiva de la cualidad pre-

\footnotetext{
${ }^{4}$ Friedman 2000 contiene una exposición sucinta de lo esencial de esta transformación; cfr. especialmente las pp. 27-28.

${ }^{5}$ Cfr. Cassirer 1910, cap. IV, § 1, subsección "Desarrollo de los conceptos de objetividad y subjetividad".
}

Diánoia, vol. LIII, no. 60 (mayo 2008). 
tendidamente objetiva que atribuimos al objeto o cosa percibido -el quale "rojo" de la propiedad de ser rojo o rojez, podríamos decir en terminología actual- (cfr. loc. cit., subsección "La serie de grados de objetividad").

Sin embargo, como sabemos, al menos algunas de estas propiedades supuestamente objetivas que atribuimos a los objetos percibidos (por ejemplo, los colores) se excluyen en una atribución científica que aplica los criterios mencionados dentro de una perspectiva más amplia, con lo que, tomando esto en consideración, parecería como si debiéramos considerar como relativamente subjetivas a tales propiedades. No obstante, su exclusión de las atribuciones científicas no implica que las mismas queden relegadas a meras apariencias subjetivas. Por el contrario, hemos de seguirlas considerando como algo "'real', aunque tenemos que concluir que esta realidad no es algo que subsista aisladamente y por sí misma, sino algo que resulta de la interacción del estímulo físico con el órgano sensorial apropiado" (loc. cit., subsección "La subjetividad de las cualidades sensibles").

En todo caso, la relativa permanencia de las cualidades sensibles (cuando se las contrasta con las impresiones) que atribuimos a las cosas de nuestra experiencia se queda corta frente al hecho de que concebimos a éstas como objetos duraderos. Dado que nuestras "tomas" de estos objetos que consideramos como duraderos son instantáneas, se requiere "el perfecto rellenado de la serie temporal". ${ }^{6} \mathrm{El}$ procedimiento general es el de "transformar y enriquecer lo dado sobre la base de la exigencia lógica de su completa conexión" (ibid.).

Así pues, para Cassirer - como para Natorp- no hay una dualidad entre sensación y pensamiento. El procedimiento que hemos visto actuar al examinar la experiencia perceptiva corriente - sostiene Cassirer - tiene su continuidad en la ciencia. En ésta, "[1] as tendencias lógicas que se encuentran en el concepto de experiencia en la concepción corriente del mundo se retoman ahora conscientemente y se llevan más allá con metódico propósito". En esta continuada aplicación del procedimiento basa la ciencia "su definición de la naturaleza y de objeto natural". Por ello, dice Cassirer, "[1] as 'cosas' de que se trata entonces resultan ser — cuanto más se comprende su significado realexpresiones metafóricas de conexiones permanentes de fenómenos de acuerdo a leyes, y por tanto expresiones de la constancia y continuidad de la experiencia misma" (ibid.).

\footnotetext{
${ }^{6}$ Loc. cit., subsección "La serie de grados de objetividad".
} 
Es así como para Cassirer y Natorp, del mismo modo en que no hay una dualidad entre sensación y pensamiento, ${ }^{7}$ no hay ninguna distinción filosóficamente relevante entre lo que les sucede al objeto de la percepción corriente y al objeto del conocimiento científico. Ambos son productos de una actividad constitutiva que se prolonga indefinidamente, pues corresponde al, en principio, ilimitado progreso del conocimiento científico que formula siempre nuevas "conexiones permanentes de fenómenos de acuerdo a leyes". La función del conocimiento empírico "no llega a ningún término en ninguna de sus actividades [...] detrás de cada solución que se le de ve una nueva tarea [...] . Aquí, de hecho, la realidad 'individual' confirma su carácter fundamental de inexhaustibilidad" (cfr. Cassirer 1910, p. 309).

Dada la falta de toda relevancia de la distinción entre objetos de la percepción corriente y objetos del conocimiento científico, y teniendo en cuenta el interés preponderante de Cassirer (como el del neokantismo de la Escuela de Marburgo en general) en éste último, no es de extrañar que el estudio de los estadios correspondientes a la percepción corriente quede en poco más que una colección de observaciones preliminares para pasar al tema considerado importante. ${ }^{8}$ En esa misma medida, el análisis neokantiano no es ulteriormente relevante para nuestros propósitos.

\section{La teoría carnapiana de la constitución en el Afbau}

Carnap se opuso explícitamente en el Afbau a la tesis neokantiana de que sólo es posible la determinación del objeto de conocimiento empírico en la serie infinita (o indefinidamente prolongada) de estructuras determinantes que se presentan con el avance científico:

De acuerdo con la concepción de la Escuela de Marburgo [...], el objeto es el eterno $X$, su determinación es una tarea incompletable. Contrariamente a esto hay que observar que un número finito de determinaciones basta para [...] su descripción unívoca entre los objetos en general. Una vez

${ }^{7}$ En la neokantiana Escuela del Sudoeste se mantiene la distinción entre la multiplicidad de la sensación considerada como preconceptual y las formas del pensamiento.

${ }^{8}$ Cassirer retomó el tema de la percepción en el tercer volumen de su obra magna, La filosofía de las formas simbólicas. Pero tampoco allí aparece la percepción como un tema focal. Cassirer está interesado primariamente en tratar el tema en relación con la expresión y el mito (parte I, capítulo 1) o en relación con transtornos del habla (parte II, capítulo 6).

Diánoia, vol. LIII, no. 60 (mayo 2008). 
que se ha dado una descripción, el objeto no es ya un $X$, sino algo unívocamente determinado - algo cuya subsiguiente descripción completa es ciertamente una tarea incompletable. (Carnap 1928, § 179)

Carnap pretende, pues, hacer posible la distinción entre dos tipos de caracterización: la determinación de cuáles son los objetos de que trata el conocimiento empírico, y la determinación de qué propiedades tienen éstos. Ciertamente, está claro que el primer tipo de determinación se sirve también de ciertas propiedades de los objetos, las cuales se han de considerar como propiedades constitutivas de los mismos. La tarea por realizar aquí es caracterizar los diversos tipos de objetos de conocimiento empírico, y es precisamente esto lo que Carnap pretende haber hecho en su mencionada obra. La segunda tarea es describir tales objetos mediante ulteriores propiedades (no constitutivas); es la tarea de la ciencia, tarea que sí ha de concebirse como indefinidamente prolongada.

Precisamente al primer tipo de tarea, la de "determinación unívoca" de los objetos, se refiere Carnap como "constitución", concebida no como investigación de un proceso psicológico real, sino como reconstrucción racional. Carnap sostiene que su teoría es neutral entre el punto de vista idealista, según el cual los objetos son "generados en el pensamiento" - punto de vista concretamente atribuible a la Escuela de Marburgo-, y el punto de vista realista, según el cual los objetos son "reconocidos en el pensamiento". La tarea definicional o "constitucional" pretende caracterizar cada tipo de objetos mediante una descripción definida que precisa los aspectos estructurales de los objetos de ese tipo. ${ }^{9}$

El objetivo general de Carnap es mostrar cómo es posible el conocimiento empírico objetivo, a pesar de que este conocimiento se origina en la experiencia subjetiva. Y Carnap cree haberlo alcanzado porque las descripciones caracterizadoras de los distintos tipos de objetos no hacen ninguna referencia a cualidades fenoménicas intrínsecas. En la

\footnotetext{
${ }^{9}$ Las definiciones que caracterizan los tipos de objetos correspondientes mediante tales descripciones las concibe Carnap como estipulaciones; es decir, por tanto, como convenciones explícitas, y pueden ser consideradas como enunciados analíticos a priori. En cambio, los enunciados que asignan propiedades no constitutivas a los objetos son enunciados sintéticos a posteriori. Así, no hay lugar para enunciados sintéticos a priori. Aunque en este importante aspecto Carnap se aparte claramente de la filosofía kantiana, es preciso reconocer que la motivación de fondo de su trabajo pertenece explícitamente a esa tradición, como ha quedado claro tras los estudios históricos de autores como Haack, Moulines, Sauer y Friedman (cfr. las referencias en Friedman 2000).
} 
realización de su proyecto, Carnap mantiene una separación importante entre lo que en escritos anteriores al Afbau denominaba el "mundo primario" de la experiencia sensorial y el "mundo secundario" de los objetos físicos. El primero está ahora incluido en el ámbito de lo autopsicológico (es decir, del psiquismo propio), y la distinción entre lo autopsicológico y lo físico es precisamente la distinción más central del Afbau. ${ }^{10}$ Además - y éste es uno de los aspectos que ofrece un interés especial desde la perspectiva de este trabajo-, se diferencia claramente entre la constitución de los objetos físicos de la percepción (las "cosas visuales") y los objetos físicos en el sentido en que son los tratados en la física.

Es bien sabido que el proyecto carnapiano del Afbau se enfrenta a dificultades internas insuperables, precisamente a partir del paso del ámbito del psiquismo propio al de los objetos físicos de la percepción, y probablemente se ve afectado además por otras críticas contundentes (como la crítica quineana dirigida al estatus o el papel de las verdades por convención). ${ }^{11}$ Para los presentes propósitos, sin embargo, tiene mayor relevancia una consideración crítica del procedimiento del cual se sirvió Carnap en su teoría sobre la constitución. Como es sabido, Carnap utilizó la teoría de los tipos (lógicos) de Principia Mathemati$c a$, de tal modo que las anteriormente aludidas descripciones de los diferentes tipos de objetos (en el sentido corriente) sitúa a éstos unívocamente en la jerarquía de tipos lógicos. Con ello, lo que efectivamente hace Carnap es modelar lógico-matemáticamente esos diferentes tipos de objetos (en el sentido corriente del término). El problema general de este procedimiento es que no está claro que ciertas propiedades de los objetos del modelo coincidan con propiedades interesantes de los objetos modelados. Así, entidades intuitivamente concebidas como reales y entidades también intuitivamente concebidas como ideales resultan ser por igual, en el modelo carnapiano, "cuasi-objetos", pero es sumamente cuestionable que esto establezca realmente - como Carnap pretendeuna neutralidad epistemológica o metafísica entre esas entidades.

\footnotetext{
${ }^{10}$ Los otros ámbitos tratados más sucintamente en esta obra son el de lo heteropsicológico (es decir, de las mentes ajenas) y el de lo intersubjetivo, que incluye los objetos culturales.

${ }^{11}$ Puede verse una exposición sucinta de la dificultad interna principal en Friedman 2000, pp. 83-85. Para otras dificultades, cfr. Moulines 1973. En el capítulo III de esa obra se encuentra una excelente exposición del sistema de Carnap, aunque la perspectiva sobre el Afbau es la empirista-fenomenalista (Moulines fue posteriormente uno de los pioneros del estudio de los elementos kantianos del proyecto carnapiano). La crítica que se esboza más adelante, por lo que yo sé, no se ha realizado hasta ahora, aunque creo que es bastante obvia.
} 
Para el propósito presente de considerar la objetivización en el contexto de la percepción, un punto concreto del procedimiento modelista carnapiano que ofrece un interés especial es que en el modelo se pierde toda base para distinguir entre objetos y conceptos. Esto es así porque casi todas las entidades ("cuasi-objetos") constituidas son clases (clases de clases, clases de clases de clases, etc.). Así, un concepto se modela extensionalmente como una clase; pero esta misma clase aparece como elemento de una clase de nivel superior, con lo cual se la trata como objeto. Esta desaparición de una base objetiva para la distinción entre objeto y concepto supone un costo excesivo del procedimiento de modelización para un proyecto de análisis de la percepción.

Hay, por último, un inconveniente ulterior insuperable que tiene que ver con el hecho de que la caracterización ("constitución") de los objetos en el Afbau se realiza enteramente, como se ha dicho ya, mediante descripciones (definidas). Debido a este rasgo, el proyecto carnapiano deja forzosamente fuera el aspecto demostrativo de la percepción, un aspecto del que sí se ocupó Husserl, como se verá en la sección final de este artículo.

\section{El problema de la constitución en la fenomenología husserliana}

En una perspectiva kantiana sobre la constitución, lo que se constituye es el objeto de la percepción. De este modo, cuando la percepción se analiza simplemente en términos de sujeto-objeto, se facilita concebir esa constitución como una especie de "construcción" del objeto y, efectivamente, ésa es a veces la terminología empleada, que encuentra una plena explicación en el contexto de una concepción idealista (o de otro modo antirrealista) de lo conocido en la percepción.

En el texto siguiente se refleja un cambio de rumbo de una gran importancia:

[L]os objetos de los que somos conscientes — dice Husserl— no están simplemente en la conciencia como en una caja, de modo que uno no puede simplemente encontrárselos en ella y "agarrarlos" (greifen) en ella, sino que primeramente se constituyen como lo que son para nosotros y como lo que cuenta para nosotros, en las diversas formas de la intención [percepción, imaginación, memoria] objetiva. ${ }^{12}$

${ }^{12} L U$, II/1, 165; LI, I, 385; IL, I, Investigación segunda, cap. 3, § 23, 338; las cursivas son de Husserl. No existe una forma estandarizada de dar la referencia de pasajes de las Investigaciones lógicas. Sigo aquí una de las maneras presentes en la literatura, en que las cifras en numeración romana no indican de cuál de 
Según el texto, los objetos de la percepción (como los de la imaginación o la memoria, pero nos limitaremos a los primeros) cuentan para nosotros como algo, son para nosotros de cierta manera, y estos modos en que cuentan, o las maneras en que son para nosotros, es de lo que se trata en (o quizá, más precavidamente: está conectado estrechamente con) la constitución.

La nueva perspectiva husserliana tiene fundamentalmente que ver con el desarrollo de una teoría explícita del contenido intencional, y en particular del contenido intencional de la percepción. El elemento decisivamente clarificador es la estructura tripartita de esta teoría: sujetocontenido (intencional)-objeto. ${ }^{13}$ Repasemos sus rasgos principales.

Se ha de advertir ante todo que, para Husserl, el objeto u objetividad percibido no forma parte constitutiva del acto o vivencia intencional (Husserl habla de "vivencia" o "acto" donde hoy día se diría "estado mental" o "estado intencional"). Husserl pretendía describir las características esenciales de los estados intencionales y piensa que el hecho de que no a todo estado mental le corresponda un objeto (lo que puede ocurrir en casos de percepción alucinatoria) descalifica al objeto como parte esencial del acto. En correspondencia con esto, el análisis fenomenológico husserliano se atiene a lo que Husserl denomina Epoché - término que tomó prestado de los antiguos escépticos, entre quienes significaba "suspensión de juicio" - o "puesta entre paréntesis" del objeto que pudiera tener el acto. Esto tiene una consecuencia inmediata sumamente importante para disipar algunas dudas que pueda haber suscitado el texto citado anteriormente y, en general, para clarificar cómo la doctrina husserliana de la constitución se aparta de las ambigüedades de la tradición kantiana: debido a que el objeto no forma

las seis investigaciones se trata, sino simplemente el volumen. Así, en referencia al original alemán $(L U)$ "II/1" indica que el pasaje se encuentra en el volumen II de la obra, en la primera de las dos subpartes de que consta este volumen. La paginación es la de la edición original, reproducida en los márgenes de la edición crítica de Husserliana. Se incluye también la referencia a la traducción inglesa ( $L I)$, bastante difundida, dando igualmente el número de volumen y la página de la edición que se cita en las referencias. Las traducciones son mías; no obstante, también doy la referencia de la traducción al español (IL).

${ }^{13} \mathrm{Al}$ decir "objeto", debe tenerse en cuenta que Husserl incluye también en su análisis experiencias perceptuales de estados de cosas, por lo que a menudo utiliza el término más general "objetividad" como término de referencia dividida: una vivencia intencional está dirigida (o es como si estuviera dirigida) a una objetividad (eine Gegenständlichkeit). Aquí podemos pensar ante todo en el caso de la percepción de objetos individuales, pero lo que se dice se aplica también mutatis mutandis a las "objetividades" en general, en el sentido indicado. 
parte del análisis fenomenológico del acto, la constitución —parte importante de ese análisis- no puede entenderse como referida al objeto. ¿A qué concierne, pues, exactamente?

Consideremos el contraste entre las experiencias que podríamos describir, respectivamente, como las de ver un pájaro negro y ver, simplemente, una cosa negra (de tamaño y forma, supongamos, similares a las del pájaro). Está claro que "lo que cuenta para nosotros", aquello que se ve (el objeto), es distinto. En la terminología de la constitución podríamos decir que lo visto ha sido "constituido" de forma distinta en un caso y otro. Ahora bien, la constitución de los objetos de nuestros estados intencionales no debe entenderse como algún tipo de construcción de los mismos en ningún sentido aceptablemente literal. Como dice muy atinadamente Føllesdal: "[N]o significa que [los objetos] sean causados por nuestros actos o producidos por nuestros actos, sino sólo que en el acto los diversos componentes de la conciencia están interconectados de modo que tenemos una experiencia de un objeto hecho y derecho" (Føllesdal 1976, p. 97).

Para entender más cabalmente la segunda parte de esta observación, preguntémonos en qué radica, más precisamente, que las dos experiencias anteriormente mencionadas sean distintas. En las Investigaciones lógicas, Husserl llama "materia" (Materie) del acto o estado intencional al elemento que lo diferencia de otros respecto de su "tema" (es decir, la percepción de un libro y la de una mesa son actos o estados con materia distinta, como lo son, por tanto, la percepción de un pájaro negro y una cosa negra indeferenciada). Con cierta simplificación puede decirse que en las obras posteriores a Ideas Husserl recoge bajo el término "noema" este mismo "aspecto temático" de las vivencias intencionales. Según la corriente interpretativa probablemente más importante de la teoría de la intencionalidad de Husserl, la iniciada por Føllesdal y desarrollada por él mismo y por autores como Dreyfus, McIntyre, Smith y Miller, entre otros, la noción de noema se entiende estrechamente relacionada con la noción fregeana de sentido, quizá como una especie de generalización de la noción fregeana a la teoría del contenido de los actos intencionales. ${ }^{14}$

${ }^{14}$ Cfr. Føllesdal 1969, Smith y McIntyre 1982 y los ensayos de Dreyfus y Miller incluidos en Dreyfus 1982. La teoría de la intencionalidad de Husserl en Ideas se explica de manera sistemática e iluminadora en los capítulos IV y V del libro de Smith y McIntyre. Con respecto a la teoría de la intencionalidad de las Investigaciones lógicas, en Künne 1986, pp. 193-198, se explica con gran claridad la estrecha relación de la noción de sentido con la de materia de un acto. Künne argumenta (loc. cit.), sin embargo, que la noción husserliana de significado de una expresión 
Para entender la doctrina husserliana de la constitución es preciso considerar la relación entre el tipo de cosa (una cuestión ontológica) y la manera "en que son para nosotros las cosas" (algo que pertenece a la caracterización de la vivencia o acto y que es lo que quedaría recogido en el noema). Respecto del primer término de esta relación, como es sabido, Husserl pensaba que es posible (y fundamental) investigar a priori dominios de objetos o "regiones ontológicas". La clave para esta investigación es considerar intuitivamente objetos representativos y examinar si atribuirles o dejar de atribuirles determinadas propiedades hace que los objetos dejen de ser del tipo que son. Siguiendo este procedimiento (el denominado método de la "variación libre") se determinarían las propiedades que son esenciales para que los objetos en cuestión sean de ese tipo o pertenezcan al dominio o región ontológica en cuestión (a esta determinación la denomina Husserl "reducción eidética"). ${ }^{15}$

En Ideas § 149 toma como ejemplo el dominio de las "cosas (materiales) en general". Esta "región ontológica" se articula, según Husserl, en diversas partes o "componentes". Aplicando el procedimiento anterior a este dominio, nos encontramos con que tales cosas se han de considerar necesariamente como objetos que duran un tiempo indefinido

lingüística (el significado de un enunciado que expresa un juicio es la propiedad semántica del enunciado que corresponde a la materia del acto intencional del juicio) es más estricta que la noción fregeana de sentido. Trasladando lo que esto implica a la noción de noema (la noción correspondiente a la de materia de un acto en la teoría de Ideas), este carácter más estricto introduce una corrección menor a la tesis de que la noción de noema es una generalización de la noción fregeana de sentido.

${ }^{15}$ Se trata de un procedimiento de determinación llevado a cabo, según Husserl, en la imaginación. De ahí que lo califique continuamente de "intuitivo" y hable de "intuición de esencias" (Wesenserschauung). Naturalmente, desde la perspectiva poskripkeana de la filosofía actual, la atribución de propiedades esenciales incluso a individuos se considera algo perfectamente admisible; sin embargo, con la excepción de determinados dominios —notablemente los de la matemática一, se pondrían fuertes reparos a la idea de que la determinación de propiedades esenciales es posible a priori. Por el contrario, se considera frecuentemente el caso de propiedades poseídas necesariamente, pero de cuya posesión sólo es posible tener conocimiento a posteriori. Por otra parte, la posible importancia de esta crítica potencial depende de la relevancia que se le haya de dar a la repetida afirmación de Husserl de que las investigaciones ontológicas mencionadas no son "metafísicas" o no forman parte de una "teoría metafísica" (cfr., v.gr., Ideas, último párrafo de $\S 149$ ). Aunque no podemos entrar aquí en esta cuestión, nótese que la incompatibilidad de las ideas de Husserl con respecto a la perspectiva mencionada quedaría eliminada si interpretáramos las esencias husserlianas en el sentido de las "esencias nominales" de Locke. 
(res temporalis), "como - dice también Husserl- en principio indefinidamente extensibles respecto de su duración" (loc. cit.). Las cosas en cuestión han de considerarse, además, como entidades con propiedades espaciales (res extensa); es decir, o bien como "susceptibles, con respecto a sus relaciones espaciales, de infinitos cambios de forma", o bien - "allí donde la configuración o el cambio de configuración permanece constante" - como susceptibles "de infinitos cambios de posición" (ibid.). Las cosas son, finalmente, res materialis; es decir, unidades sustanciales que, como tales, entran en "conexiones causales, infinitamente variadas en sus posibles estructuras" (ibid.; las cursivas son del propio Husserl); en definitiva, podríamos decir, cosas materiales propiamente dichas. ${ }^{16}$

El procedimiento de determinación de las propiedades esenciales (la "reducción eidética") se aplica también a las vivencias o actos intencionales (precisamente la fenomenología en la concepción de Husserl consiste centralmente en la investigación de las propiedades esenciales de esos actos o vivencias por el procedimiento de "reducción eidética"). En particular se aplica a las vivencias o actos intencionales cuyos objetos son cosas (materiales) (cfr. § 150). Aquí, lo que centralmente ha de reconocerse es que las apariencias de las cosas — "noemáticamente entendidas", dice Husserl; es decir, considerando los noemata bajo los cuales se nos presentan las cosas-, aunque infinitamente variables, no son arbitrariamente variables. Así, en el caso de la percepción de un objeto material particular de un tipo determinado, la multiplicidad de apariencias en que ese objeto puede presentarse (por contraste, por ejemplo, con la multiplicidad de apariencias con las que un objeto de diferente tipo puede presentarse) no constituye una unidad accidental; al contrario, se trataría de una serie de apariencias "completamente determinada, ordenada de una manera definida", que muestra "una organización interna definida de sus modos de desarrollo" (loc. cit.).

La regulación de las series de apariencias de cosas (materiales, en sentido general) sigue pues, de algún modo, la esencia correspondiente a tales cosas. Husserl lo expresa diciendo que esa esencia regional "prescribe reglas para las multiplicidades de apariencias" (ibid.; las cursivas son del original). Sin embargo, es crucial observar que este "prescribir"

${ }^{16}$ Más allá de la región ontológica de las "cosas (materiales) en general”, la formación inmediatamente superior es la de las cosas intersubjetivas (Ideas, § 151) y, por encima, menciona Husserl a las "comunidades intersubjetivas", los "objetos poseedores de valores", los “objetos prácticos" y "las organizaciones culturales concretas" (el Estado, la Iglesia y la ley son ejemplos de esta últimas que da el propio Husserl, cfr. Ideas § 152). 
ha de interpretarse de manera consistente con la afirmación de Husserl de que las conexiones entre las fenomenologías constitutivas y las correspondientes ontologías no ha de entenderse en absoluto como "una fundamentación de las primeras en las segundas" (Ideas, § 153; las cursivas son del original). Más bien lo que ocurre es que una proposición o un concepto ontológico puede servir "como indicador de conexiones constitutivas y esenciales", especialmente entre distintos noemata de actos intencionales; pero aunque ese concepto o proposición puede, en este sentido, ser una clave para llegar a establecer tales conexiones, éstas "llevan su autoridad y su validez en sí mismas" (loc. cit.).

Debido a la mencionada prescripción, la "organización interna definida" de los modos de desarrollo de una serie de apariencias correspondiente, por ejemplo, a la percepción de un objeto individual ha de ser "consistente con las [esencias] designadas de un modo general como componentes de la [esencia] regional de las cosas consideradas en general" (ibid.). Es decir, por ejemplo, que dicha organización es tal que la unidad de las apariencias correspondientes a "una mera res extensa es concebible aparte de la unidad regulada por [la esencia de] la res materialis" (es decir, por la esencia de las cosas materiales en sentido estricto o propio), pero no a la inversa. ${ }^{17}$

De este modo, las diversas unidades de apariencias —vale decir, los diversos noemata- se sitúan en diferentes estratos. Husserl expone claramente esta idea en el volumen I de Ideas y la desarrolla en el volumen III, donde se concentran sus investigaciones detalladas sobre la teoría de la constitución. Sin embargo, la conclusión que realmente nos interesa es que, se entienda como se entienda concretamente la regulación de las "unidades" aludidas por las diversas esencias regionales, la cuestión de la constitución concierne propiamente a los noemata de los actos o vivencias intencionales, no a los objetos de tales actos. Complementamos así en este contexto más específico la conclusión a la que habíamos llegado de manera general al principio, a partir de la

${ }^{17}$ Naturalmente, también las vivencias o actos intencionales fundamentalmente asociados a las "entidades objetivas" (Objektivitäten) de orden superior mencionadas en la nota precedente deben ser investigadas desde el punto de vista de la constitución, aunque los actos intencionales fundamentalmente asociados a ellas no serían actos de percepción, sino otras clases de actos (por ejemplo, los actos que se corresponden con las "cosas intersubjetivas" son actos de empatía; cfr. Ideas $\S 151)$. Todos esos actos deben también ser descritos fenomenológicamente; es decir, "en el modo en que [las correspondientes entidades] vienen a ser presentadas de acuerdo con sus tipos fundamentales y en su adecuado orden de formación" y "los problemas de constitución fenomenológica se han de formular y resolver en su caso" (Ideas, § 152).

Diánoia, vol. LIII, no. 60 (mayo 2008). 
no pertenencia del objeto al acto intencional según el análisis que de los estados intencionales hace la fenomenología husserliana.

\section{La desaparición del problema de la constitución}

Si atendemos al panorama filosófico actual, difícilmente encontraremos algún escrito filosófico que trate del problema de la constitución. Esta situación no es debida, desde luego, a que el problema haya sido definitivamente resuelto (algo que en filosofía podemos siempre o casi siempre descartar), por lo que se podría hablar de pérdida de vigencia del problema. En realidad, la situación es algo más compleja, pues, como veremos, más bien deberíamos hablar de aparente pérdida de vigencia.

Las causas de la situación actual del problema de la constitución son muy diversas y seguirles la pista conduciría a repasar buena parte de la historia de la filosofía del siglo xx. Sin embargo, su identificación es relativamente fácil y pueden al menos darse en forma de lista, aunque, desde luego, no sea posible penetrar en su explicación.

Empezaremos con las causas de la desaparición del problema en cada una de las escuelas o tendencias filosóficas de principios del siglo xx que hemos tratado: neokantismo, filosofía analítica y fenomenología.

Respecto del primero, la situación es clara: la desaparición del problema coincide con la desaparición del neokantismo como tendencia filosófica reconocible. A su vez, las causas de la desaparición del neokantismo serían básicamente dos: dificultades por acomodar las revoluciones de la física (teoría de la relatividad y mecánica cuántica) y factores sociológicos. La primera podría tal vez discutirse. ${ }^{18}$ Los segundos se encuentran en los dramáticos cambios en la filosofía universitaria que tuvieron lugar en Alemania como resultado de la llegada al poder del nacionalsocialismo, y que llevaron, por ejemplo, al último gran representante del neokantismo - Cassirer - al exilio (donde el hecho de que falleciera transcurridos no muchos años contribuyó en gran medida a que su orientación filosófica no pudiera echar hondas raíces).

Los avatares del problema de la constitución en la tradición analítica son aún más complejos. Por limitarnos, de momento, a quien sin duda fue durante unos años el "portaestandarte" del problema, la filosofía de Carnap había de sufrir una transformación muy importante poco después de la publicación del Aufbau, como resultado de las discusiones

${ }^{18}$ Michael Friedman ha tratado de articular una epistemología de la física y de las ciencias más en general que tal vez sería posible considerar — según él mismo sostiene- como heredera de ciertos rasgos de la filosofía de Cassirer. Véase Friedman 2001. 
filosóficas entre los miembros del Círculo de Viena. Muy concretamente, Carnap abandonó todo intento de elaborar una epistemología sobre la base de la experiencia subjetiva.

Por lo que concierne a la fenomenología, debe tenerse en cuenta el proceso de sustitución del predominio de la fenomenología husserliana por la fenomenología existencial y, más tarde, por la filosofía hermenéutica. Los factores sociológicos mencionados en relación con el neokantismo desempeñaron también aquí un papel importante. Sin embargo, las causas digamos "internas" son también interesantes. El problema de la constitución no desaparece en la fenomenología existencial de Heidegger (véase, por ejemplo, Føllesdal 1979); pero, a más largo plazo, el "giro" del pensamiento de Heidegger y la gran influencia del mismo habían de resultarle fatales.

Volvamos ahora a Carnap para trazar sucintamente los desarrollos relevantes en la tradición analítica posterior. Carnap sustituyó su proyecto epistemológico basado en la experiencia subjetiva por el fisicismo; es decir, en su caso, por la adopción de un "lenguaje fisicista", un lenguaje en el que se habla de objetos macroscópicos y de sus propiedades, sin intentar "construir" o "constituir" tales objetos desde una base en el "psiquismo propio". Sin embargo, oficialmente Carnap no renunció a la idea de que la elección entre "lenguajes" (o marcos conceptuales) - $\mathrm{y}$, en particular, la elección entre un "lenguaje fisicista" y un "lenguaje fenomenalista" - sólo se podía hacer por motivos pragmáticos, no por razones teóricas. La influyente crítica de Quine a éste y otros aspectos del proyecto carnapiano hicieron por un largo tiempo aún más remota la posibilidad de retomar el problema de la constitución. Además, la influyente filosofía quineana tuvo también un efecto negativo más directo por su oposición a toda forma de "mentalismo" y, en especial, a cualquier noción de contenido intencional.

La disminución de la influencia de la filosofía de Quine vino emparejada con desarrollos que en nada favorecieron la atención de los filósofos al problema de la constitución: la mayor influencia, en general, de la filosofía wittgensteiniana, la crítica al fundamentismo empirista en epistemología y, en filosofía del lenguaje y de la mente, el predominio de "teorías de la referencia directa" y el externismo acerca del contenido semántico de los enunciados o el contenido intencional de los estados mentales. Concentrando nuestra atención en el último aspecto, este tipo de externismo, emparentado con el externismo sobre el contenido semántico propugnado por autores tan influyentes como Kripke, Putnam y Kaplan, no es en sí mismo opuesto a toda forma de "mentalismo", pero sí parece ser incompatible con rasgos centrales de la 
teoría de la intencionalidad de Husserl en la que, como se ha mostrado, el problema de la constitución encontró un mayor y mejor desarrollo. Recordemos en particular, una vez más, que Husserl pensaba que el propio objeto "referido" no es parte esencial del estado mental.

Todos los reseñados son factores muy importantes que explican la situación actual respecto del problema de la constitución; sin embargo hay un último factor, digamos inesperado, que presenta la cuestión bajo una nueva luz. Merece que le dediquemos un apartado.

\section{El caso del problema camuflado}

Lo que quisiera sostener, por último, es que la pérdida de vigencia del problema de la constitución es, en gran parte, sólo aparente: el hecho de que, contemplando el panorama filosófico actual, no pueda "vérsele" se debe a que está, por así decir, camuflado. Camuflado sobre todo bajo una terminología diferente. Para defender esta afirmación procederé a dar una breve explicación, una ilustración y un pequeño argumento.

El problema de la constitución, según lo hemos caracterizado en el contexto finalmente más relevante, el de la fenomenología, es el de explicar los diversos modos en que la conciencia "se dirige" (o es como si "se dirigiera") a un objeto. En particular, puede ser así descrito en el contexto del análisis de la percepción. Pues bien, no es otra cosa lo que se trata de dilucidar en una de las corrientes más importantes de la filosofía analítica actual: el "fregeanismo en sentido amplio" o "neofregeanismo" de autores tan influyentes como Evans, McDowell y Peacocke, entre muchos otros, cuando hablan del "modo de determinación" o "modo de presentación" de los objetos. Contemplados desde una perspectiva general, tales "modos de presentación" no son en ese neofregeanismo sino una especie de generalización de la idea fregeana de "modo de darse" el referente de una expresión lingüística. Es pertinente, por tanto, recordar que, según habíamos visto, la noción husserliana de noema podía, con algunos matices, considerarse también como una tal generalización. ${ }^{19}$

\footnotetext{
${ }^{19}$ La afinidad — temática y de enfoque- de los autores de la corriente neofregeana con Husserl no le ha pasado desapercibida a Dummett, sin duda uno de los mayores expertos en Frege. Dummett llega a excluir a autores como los mencionados - en particular a Evans - del campo de la corriente analítica debido a que renuncian a que "una explicación filosófica del pensamiento pueda conseguirse por medio de una explicación filosófica del lenguaje" (Dummett 1994, p. 4). Precisamente lo que se pretende en el presente trabajo es mostrar la relevancia
} 
Como ilustración del hecho de que no estamos aquí ante ninguna coincidencia casual, consideremos brevemente un aspecto reciente de la filosofía de la percepción.

En los últimos años, diversos autores -entre ellos: Evans, Searle, McDowell, Burge, Brewer, Campbell-, casi todos ellos identificados con el mencionado fregeanismo en sentido amplio, han insistido en lo que puede denominarse el carácter demostrativo de la percepción, tratando la mayoría de ellos de proporcionar una explicación filosófica de en qué consiste este rasgo central de la percepción. Pues bien, Husserl mismo había anticipado dicho rasgo, como han enfatizado —de manera prácticamente independiente de los autores anteriormente nombrados- intérpretes recientes como Miller (1984) y D.W. Smith (1984). Se trata en parte de que, dicho preanalíticamente, en casos muy característicos de percepción (especialmente en casos de percepción visual, pero con seguridad no sólo de ella), el objeto se presenta, podríamos decir, "directamente", con una inmediatez característica. ${ }^{20}$ Aplicando la teoría de la intencionalidad husserliana a un análisis de dicho rasgo, se trataría de caracterizar el llamado por Husserl componente " $X$ ", o componente no descriptivo del noema del acto de percepción. Aunque no es posible entrar aquí en detalles, los esfuerzos de algunos de los autores analíticos mencionados, o de los autores neohusserlianos aludidos, son claramente esfuerzos por solucionar exactamente el mismo problema y, en algunos casos al menos, por vías no muy divergentes. Al fin y al cabo, de lo que se trata es de describir, en el caso de la percepción, aquello en la experiencia que la caracteriza como "conciencia inmediata" de un objeto individual (confróntese esta observación con la breve explicación de la noción de constitución de Føllesdal que se citó en la sección 4). ${ }^{21}$

de esta transformación para el problema de la constitución, que Dummett no trata explícitamente.

${ }^{20}$ En cierto modo, esta inmediatez fue reconocida en la noción russelliana de acquaintance, pero Russell pensó que el "contacto directo" en la percepción se da con datos sensoriales. En la influyente obra de Evans Varieties of Reference se hace una defensa, desde la perspectiva neofregeana, de la noción de acquaintance, desprovista del internismo russelliano (es decir, el "contacto directo" se da, en los casos paradigmáticos, con un objeto físico).

${ }^{21}$ Es cierto que buena parte de la discusión se ha centrado principalmente en la "conciencia inmediata" que nos proporciona la percepción de propiedades básicas de los objetos —así en McDowell 1994 y en la discusión relacionada en torno a la distinción entre contenido conceptual y contenido no conceptual-. Pero esto no es cierto respecto de Evans 1982, la obra a la que puede atribuirse el inicio de la discusión en el campo neofregeano, ni de trabajos recientes como Campbell 2002. 
Puedo anticipar una objeción a la tesis que estoy sosteniendo. Podría decirse que si admitimos que el problema de la constitución se presenta con claridad en primer lugar en la fenomenología husserliana, hay un rasgo diferenciador del análisis de Husserl que invalida la afirmación de que en la corriente neofregeana se trata el mismo problema con terminología algo distinta. El rasgo en cuestión es la correspondencia entre regiones ontológicas y "unidades" de articulación de la experiencia en el plano noemático. La corriente neofregeana —seguiría la objeciónestá completamente alejada de la idea de que es posible y necesaria la investigación a priori de "regiones ontológicas" y de que es legítimo el procedimiento husserliano de la "intuición de esencias". Pero, por la correspondencia mencionada, este aspecto habría de ser considerado esencial al problema de la constitución. De manera que en la corriente neofregeana, aunque puedan señalarse algunos puntos de contacto con la fenomenología husserliana, no se trata en realidad el problema de la constitución (no es sólo que no se utilice una terminología a tal efecto).

Podría tal vez replicarse que la cuestión es más bien verbal, que podemos estipular un uso del término "constitución", emparentado con el de Husserl, para abordar cuestiones relativas a los "modos de presentación" sin incluir especulaciones acerca de "correspondencias" con "regiones ontológicas". Pero creo que esta réplica ni es satisfactoria ni capta realmente la situación. Hay otra mucho más contundente. En efecto, lo que esa réplica pasa por alto, como pasa por alto la objeción misma, es que el propio Husserl no consideró esencial la cuestión de la correspondencia para una teoría de la constitución. Basta recordar un rasgo de la doctrina husserliana que se destacó en la sección 4: para Husserl, aunque los conceptos y proposiciones ontológicas pueden ser una clave para establecer las conexiones constitutivas de o entre los diversos noemata, tales conexiones "llevan su autoridad y validez en sí mismas" (Ideas, § 153).

Una cuestión clave que se plantea en las dos tradiciones o corrientes de que estamos hablando puede formularse así: "¿cuál es la relación entre un noema (tradición husserliana) o un modo de presentación demostrativo (corriente neofregeana) y un concepto?" Esta cuestión nos conduce a las discusiones recientes sobre el contenido conceptual y el contenido no conceptual de la percepción. Proseguirla nos llevaría a abordar de nuevos modos la vieja cuestión kantiana de hasta qué punto nuestras capacidades conceptuales configuran el contenido de la per-

En Fernández Prat 2006 se presenta mi propio intento de solucionar el problema mencionado en el texto. Se encontrarán ahí ulteriores referencias relevantes a los autores de la tradición analítica que lo han tratado. 
cepción. ${ }^{22}$ Es decir, dado que, indudablemente, la adquisición de conceptos aumenta nuestra capacidad de obtener información del entorno mediante o a partir de la percepción, ¿hasta qué punto se trata aquí simplemente de que se infieren nuevas creencias a partir de un contenido perceptivo aproximadamente estable, o se trata de que el contenido mismo de la percepción se altera con la adquisición de nuevos conceptos (los nuevos conceptos entran, por así decir, en el contenido de la percepción), o hay quizá una tercera alternativa en juego? Es conocida una línea de respuesta empírica a esta cuestión que viene de las hipótesis sobre la modularidad de la mente. Pero hay en ella una cuestión conceptual o constitutiva que tiene un interés filosófico más directo, y es razonable esperar que, en el trabajo en torno a esta problemática, ideas provenientes de la tradición husserliana y de la corriente neofregeana -e incluso también algunas de la tradición kantiana- puedan interactuar fructíferamente.

\section{BIBLIOGRAFÍA}

Allison, Henry, 2004, Kant's Transcendental Idealism, Yale University Press, New Haven, edición revisada y ampliada.

Campbell, John, 2002, Reference and Consciousness, Clarendon Press, Oxford.

Cassirer, Ernst, 1910, Substanzbegriff und Funktionsbegrif: Untersuchungen über die Grundfragen der Erkenntniskritik, Bruno Cassirer, Berlín.

Carnap, Rudolf, 1928, Der logische Aufbau del Welt, Weltkreis, Berlín; 2a. ed., Meiner, Hamburgo, 1961.

Dreyfus, Hubert (comp.), 1982, Husserl, Intentionality, and Cognitive Science, The MIT Press, Cambridge, Mass.

Dummett, Michael, 1994, Origins of Analytical Philosophy, Harvard University Press, Cambridge.

Evans, Gareth, 1982, The Varieties of Reference, Clarendon Press, Oxford.

Fernández Prat, Olga, 2006, "Particularity and Reflexivity in the Intentional Content of Perception", Theoria, vol. 56, pp. 133-45.

Føllesdal, Dagfin, 1979, "Husserl and Heidegger on the Role of Actions in the Constitution of the World", en Esa Saarinen y otros (comps.), Essays in Honour of Jaakko Hintikka, Reidel, Dordrecht, pp. 365-378.

—_, 1976, "Husserl's Theory of Perception", Ajatus, vol. 36, pp. 95-105.

_, 1969 , "Husserl's Notion of Noema", reimpreso en Dreyfus 1982, pp. 7380.

Friedman, Michael, 2001, Dynamics of Reason, Stanford University Press, Stanford.

${ }^{22}$ Speaks (2005) señala la conexión, aunque es escéptico acerca de los "nuevos modos".

Diánoia, vol. LIII, no. 60 (mayo 2008). 
Friedman, Michael, 2000, A Parting of the Ways: Carnap, Cassirer, and Heidegger, Open Court, Chicago.

Husserl, Edmund, (póstumo), Ideen zu einer reinen Phänomenologie und Phänomenologischen Philosophie, vol. III (Husserliana V), Nijhoff, La Haya, 1959.

— 1913, Ideen zu einer reinen Phänomenologie und Phänomenologischen Philosophie, vol. I (Husserliana III), Nijhoff, La Haya. Versión en castellano: Ideas relativas a una fenomenología pura y una filosofía fenomenológica, trad. José Gaos; reedición en Fondo de Cultura Económica, Madrid, 1985.

—, 1900-1901, Logische Untersuchungen (Husserliana XIX/1 y 2), La Haya: Nijhoff. La edición en la serie Husserliana es de la segunda edición revisada, originalmente años 1913 y 1921, pero tiene las variantes de la primera. Versión en inglés (a partir de la segunda edición): Logical Investigations, 2 vols.; reedición en Humanity Books, Nueva York, 2000. Versión en castellano: Investigaciones lógicas, 2 vols., trad. G. Morente y J. Gaos; reedición en Alianza Universidad, Madrid, 1982.

Künne, Wolfgang, 1986, "Husserl: Intentionalität", en Josef Speck (comp.), Grundproblema der großen Philosophen: Philosophie der Neuzeit IV', Vandenhoeck und Ruprecht, Gotinga.

Miller, Izchak, 1984, "Perceptual Reference", Synthese, vol. 61, pp. 35-59.

McDowell, John (1994), Mind and World, Harvard University Press, Cambridge, Mass.

Moulines, C. Ulises, 1973, La estructura del mundo sensible, Ariel, Barcelona.

Sellars, Wilfrid, 1978, “The Role of Imagination in Kant's Theory of Experience”, en Henry W. Johnstone Jr., Categories: A Colloquium, Pennsylvania State University Press, University Park, pp. 231-245.

— 1968, Science and Metaphysics: Variations on Kantian Themes, Routledge and Kegan Paul, Londres.

Smith, David Woodruff, 1984, "Content and Context of Perception", Synthese, vol. 61, pp. 61-87.

Smith, David Woodruff y Ronald McIntyre, 1982, Husserl and Intentionality: A Study of Mind, Meaning, and Language, Reidel, Dordrecht.

Speaks, Jeff, 2005, "Is There a Problem about Nonconceptual Content?", The Philosophical Review, vol. 114, pp. 359-398.

Strawson, Peter F., 1970, "Imagination and Perception", reimpreso en Freedom and Resentment and Other Essays, Methuen, Londres, 1974, pp. 45-65.

Wittgenstein, Ludwig, 1968, Philosophical Investigations, Blackwell, Oxford (1a. ed.: 1953); versión en castellano: Investigaciones filosóficas, trad. Alfonso García Suárez y Ulises Moulines, UNAM/Crítica, México/Barcelona, 1988.

Young, J. Michael, 1988, “Kant's View of Imagination”, Kant-Studien, vol. 79, pp. 140-164.

Recibido el 15 de marzo de 2007; aceptado el 21 de noviembre de 2007. 